\section{Number of Neutrons Liberated in the Nuclear Fission of Uranium}

RECENT experiments have shown that neutrons are liberated in the nuclear fission of uranium induced by slow neutron bombardment: secondary neutrons have been observed which show spatial ${ }^{1}$, energetic $^{2}$ or temporal ${ }^{3}$ properties different from those which primary neutrons possess or may acquire. Such observations give no information on the mean number of neutrons produced per nucleus split ; this number $\vee$ may be very small (less than 1) and the result of the experiment will still be positive.

We are now able to give information on the value of $v$. Let us consider the curve representing the density distribution of neutrons slowed down in an aqueous solution surrounding a primary neutron source $^{1}$; the area $S$ of this curve is proportional to $Q . \tau, Q$ being the number of neutrons per second emitted by the source or formed in the solution, and $\tau$ the mean time a neutron spends in the solution before being captured. Assuming that the solution contains only nuclei which absorb neutrons according to the $1 / v$ law (the only exception to this rule will presently be dealt with), $\tau$ is proportional to $1 / \Sigma c_{i} \sigma_{i}$, where $c_{i}$ is the concentration (atom grams per litre) of an absorbing nucleus, $\sigma_{i}$ its cross-section for the capture of neutrons of velocity 1 and the index $i$ is extended to all kinds of neutron-absorbing reactions attributable to nuclei present in the solution. Substituting the symbol $A_{i}$ for $c_{i} \sigma_{i}$ and $A_{\text {tot }}$ for $\Sigma A_{i}$, we have identically :

$$
\frac{\Delta S}{S}=\frac{\Delta Q}{Q}-\frac{\Delta A_{t o t}}{A_{t o t}},
$$

neglecting all terms of higher orders, such as those containing $(\triangle Q)^{2}, \triangle Q . \triangle A_{\text {tot }}$, etc.

Let the symbol $\triangle$ stand for the differences observed between the two solutions (uranyl and ammonium) used in our previous experiment ${ }^{1}$. Neglecting $\triangle A_{\text {tot }}$ before $A_{\text {tot }}$ introduces an ambiguity in the definition of $A_{\text {tot }}$ (uranyl vs. ammonium value) which is numerically unimportant and can be reduced by adopting the arithmetical mean $\left(A_{\text {tot }}(\mathrm{amm})+.\triangle A_{\text {tot }}\right) / 2$.

In the quantity $\triangle A_{\text {tot }}$ the uranium nuclei are represented by several separate terms standing for the different modes of neutron capture (see below); let $A_{f}$ be the term for the capture leading to fission. Every neutron has the probability $A_{f} / A_{t o t}$ of causing a fission and, since one individual fission process liberates $v$ neutrons on the average, the total number $\triangle Q$ of neutrons thus created is $Q \cdot \frac{A_{f}}{A_{t o t}} \cdot \nu$, and the equation (1) can be rewritten as follows :

$$
\nu=\frac{\Delta S}{S} \cdot \frac{A_{t o t}}{A_{f}}+\frac{\Delta A_{t o t}}{A_{f}} .
$$

Let us estimate the values of all quantities necessary to calculate $v$ according to this formula. The area variation $\Delta S / S$ can be read from the graph given in our previous letter with an error of less than 20 per cent (due to the uncertainties of inter- and extrapolation; in order to facilitate the latter, we added to the curves a further experimental point for $r=29 \mathrm{~cm}$.). The value of $A_{t o t}$ for the ammonium solution can be easily calculated from the known concentrations and capture cross-sections (hydrogen, nitrogen and oxygen). $A_{f}$ is equal to $c_{\mathrm{U}}$ ( 1.6 in our experiment), multiplied by the value of $\sigma_{f}$ given in a recent paper by Anderson et al. ${ }^{4} . \quad \triangle A_{\text {tot }}$ contains a term expressing the small difference of the hydrogen content between the two solutions; and three terms relative to uranium, namely, the fission term $A_{f}$, already dealt with, the thermal capture term $A_{c t}$ which can be calculated by using a recently found value for $\sigma_{c t}{ }^{5}$ and finally the resonance capture term $A_{r}$ which requires some explanation.

Our reasoning assumed that all neutrons introduced into the solution spend practically all their life, and are absorbed, in the thermal state. This is true in so far as the $1 / v$ law is valid for absorption of neutrons in all nuclei concerned; and, therefore, not wholly true for uranium, which shows a pronounced resonance capture of neutrons of about 25 volts $^{6}$. A certain proportion of neutrons entering the solution is bound to come within this resonance band and to be absorbed by resonance; therefore, it will never reach the thermal state. This proportion depends on the width of the resonance band and on the concentration $c_{U}$; its value in our system of symbols is equal to $A_{r} / A_{\text {tot }}$ and was numerically determined by an experiment reported elsewher $\theta^{5}$.

Putting all numerical values in the formula (2) (with $10^{-24} \mathrm{~cm}^{2}$ as the unit of cross-section), that is : $\triangle S / S=0.05 \pm 0.01 ; A_{t o t}=36 \pm 3 ; A_{f}=1 \cdot 6 \times 2=3 \cdot 2 ;$ $\triangle A_{\text {tot }}=8 \cdot 7 \pm 1 \cdot 4$ decomposable into $\triangle A_{\mathrm{H}}=1 \cdot 2 \pm 0 \cdot 1$, $A_{c t}=1 \cdot 6 \times(1 \cdot 3 \pm 0 \cdot 45)=2 \cdot 1 \pm 0 \cdot 7, A_{r}=6 \cdot 4 \pm 1 \cdot 1$ and $A_{f}=3 \cdot 2$, we find :

$$
\nu=3 \cdot 5 \pm 0 \cdot 7 \text {. }
$$

We were not able to allow for an error in $A_{f}$, since the value of $\sigma_{f}$ given by Anderson et al. contains no indication of probable error. Any error in $\sigma_{f}$ will affect $\nu-1$ in an inversely proportional way; in any case $v$ will remain greater than 1 .

The interest of the phenomenon discussed here as a means of producing a chain of nuclear reactions was already mentioned in our previous letter. Some further conclusions can now be drawn from the results reported here. Let us imagine a medium containing only uranium and nuclei the total neutron absorption of which, as compared to that of uranium, may be neglected (containing, for example, only some hydrogen for slowing down purposes). In such a medium, if $\frac{A_{f}}{A_{\text {tot }}} \cdot \nu>1 \quad\left(A_{t o t}\right.$ includes now only uranium terms), the fission chain will perpetuate itself and break up only after reaching the walls limiting the medium. Our experimental results show that this condition will most probably be satisfied (the quantity $\frac{A_{f}}{A_{t o t}} \cdot v-1$, though positive, will be, however, small), especially if one keeps in view that the term $A_{r}$, because of the self-reversal of the resonance absorption line, increases much more slowly than the other uranium terms when the uranium content of the medium is increased.

H. von Halban, JUN.

F. JoLIOT.

L. Kowarski.

Laboratoire de Chimie Nucléaire, College de France, Paris.

April 7. 